\title{
An Experimental Study of Installing-Holes Parameter Optimization for Metallic Inserts Bonded into CFRP Laminates
}

\author{
Jing Liu", a Xuemei Chen ${ }^{2, ~ b}$, Hui Zhang ${ }^{1, c}$, Jing $\mathrm{Xu}^{2, d}$, Fengtao $\mathrm{He}^{1, \mathrm{e}}$, \\ Kun Hou ${ }^{1, f}$, Zhenbo Yin ${ }^{1, g}$ \\ ${ }^{1}$ China Aero-Polytechnology Establishment, Beijing 100028, China \\ ${ }^{2}$ Chengdu Aircraft Industrial (Group) Co., Ltd, Chengdu 610000, China

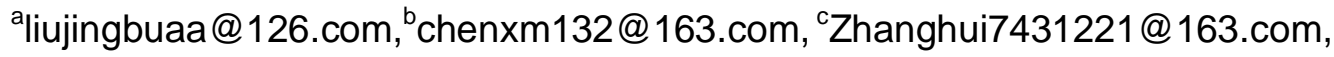 \\ djinnee.xu@gmail.com, ${ }^{e}$ hftty2006@21cn.com,thoukunbj@sina.com, ${ }^{9} 050514113 @ 163 . c o m$
}

\begin{abstract}
Keywords: installing-holes; metallic inserts; bonded; CFRP laminates
Abstract. CFRP laminates are used for various aircraft structural components because of their good mechanical and physical properties. Metallic inserts are one kind of aerospace fasteners, which are usually installed in the metallic components by an interference fit. However, when metallic inserts are installed in the CFRP laminates by the traditional installing way, delamination and low efficiencies are troublesome. Therefore, excellent quality and cost effective installing metallic inserts into the CFRP laminates remains a challenge. In this paper, a series of experiments were carried out to study the installing-holes parameter optimization for metallic inserts bonded into the CFRP laminates. The experimental results show that the push-out values and breakaway torque values of metallic inserts bonded in the CFRP laminates are not reduced obviously for 4 different type installing-holes.
\end{abstract}

\section{Introduction}

Being an advanced material, CFRP laminates are characterized by high specific strength, high specific stiffness and good corrosion resistance, which have been widely used in manufacturing of aeronautics structure components. Meanwhile, high rigidity and brittleness of CFRP laminates bring about new technical problems and difficulties in assembling. Especially for metallic inserts, the traditional interference fit is not adapted to these new types of materials which would easily lead to the stress concentration and delamination at the fastener hole of the CFRP laminates. This results in reduced strength against fatigue and compromised structure integrity [1-3]. Therefore, it is important to develop new techniques to improve the quality and efficiency of mechanically fastened metallic inserts in CFRP laminates. Over the years, some researchers have studied the bonding to fasten the metallic inserts in CFRP laminates, the method of which is considered as a good way to satisfy the joint strength and avoid the assembly defect [3-5]. In this paper, a series of experiments were performed to study the installing-holes parameter optimization for metallic insert bonded in CFRP laminates.

\section{Connection mechanism of metallic inserts bonded in CFRP laminates}

Metallic inserts are usually installed in the metallic components by interference fit shown as Figure,1(a), in which method the metallic inserts are assembled for avoiding axial and radial distance by the way of adjusting the interference amount to create friction between the outer of metallic insert and the inner of fastener hole. Therefore, the mechanical prosperity of installed joins is affected largely by the magnitude of interference between the metallic inserts and fastener holes. Shown as Figure.1 (b), compared to the interference fit, the bonding is considered for the following advantages. Firstly, the contact areas are changed for the bonded metallic inserts: the radial tractions due to the fastener-hole contact are transferred using the whole surface of the composite's hole instead of approximately half of the surface of the hole. The use of bonded metallic inserts change the failure mode from a bearing to a tension-bear mode, which is redistributes the radial stresses around the hole boundary and consequently reduce the high stress concentrations occurring 
in the bearing plane. Secondly, the nature of fasten force is changed from friction forces of the interference fit to adhere forces of bonding method. According to some studies, the adhesive forces consist of hydrogen bond, chemical bond, intermolecular forces. Therefore, the adhesive forces show good aircraft fuel resistance and have more joint strengths than that of the physical fastening method under certain conditions [3-5].

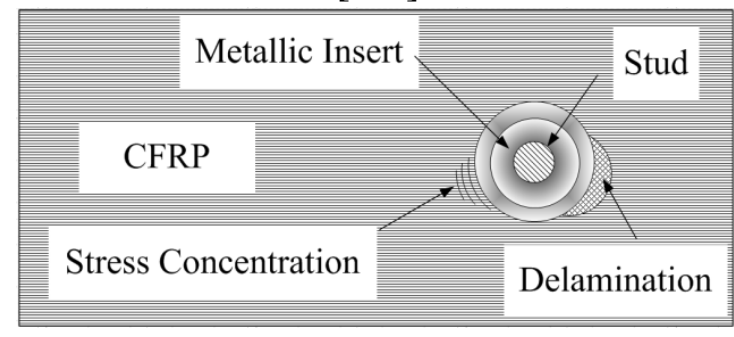

(a) Interference-fit

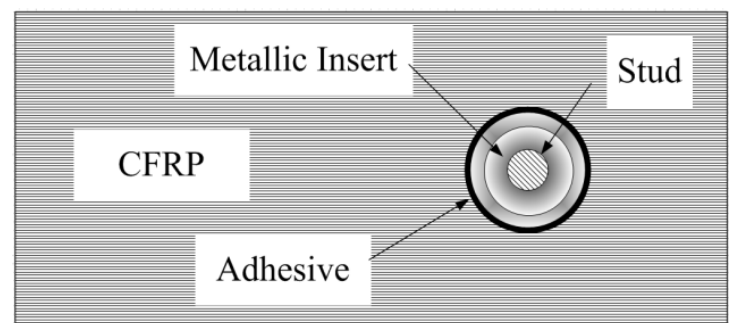

(b) Bonding

Figure.1. Comparison of the different installing methods of metallic inserts

\section{Experimental setup and procedure}

Setup and conditions. The CFRP laminates bonded with metallic inserts are showed in Figure .2 and there are two metallic inserts in each sample. The test parameters for the experiments are summarized in Table 1.Figure.3 (a) shows a torsion testing machine, which is used to test the breakaway torque values of metallic inserts installed in the CFRP laminates and mainly consists of a torsion spindle, a samples fixture, a feed direction worktable, a power supply and a computer. The maximum torque and spindle speed are 50N.m and 500rpm, respectively. Figure.4 (a) shows a computer-controlled electronic universal testing machine, which is used to test the push-out values of metallic inserts installed in the CFRP laminates and mainly consists of a loading spindle, a samples fixture, a worktable, a power supply and a computer. The maximum loading and feed speed are $20 \mathrm{kN}$ and $500 \mathrm{~mm} / \mathrm{min}$, respectively.

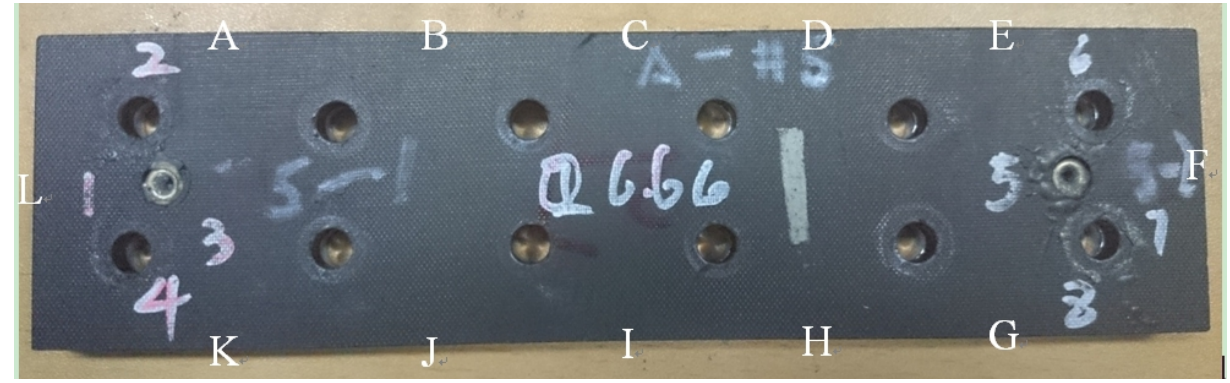

Figure.2. Sample of CFRP laminate bonded with metallic inserts

Table 1 Test parameters for the experiments.

\begin{tabular}{cc}
\hline Parameters & Sample \\
\hline outer diameter of metallic insert & $\emptyset 3 \mathrm{~mm}$ \\
Material of metallic insert & Steel \\
Thickness of CFRP laminate & $5 \mathrm{~mm}$ \\
Adhesives & Epoxy Resin Adhesive \\
Material of mandrel & $\mathrm{A} 286$ \\
Installing-holes Type & $\mathrm{A}<\mathrm{B}<\mathrm{C}<\mathrm{D}$ \\
Injection Pressure & $(0 / 60 / 80 / 100) \mathrm{PSI}$ \\
\hline
\end{tabular}

Experimental procedure. Four type installing-holes samples are prepared to test the push-out and breakaway torque values of metallic inserts bonded into CFRP laminates. Firstly, each sample are prepared to finishing injection pressure for 0/60/80/100PSI. Secondly, the push-out and breakaway torque tests are carried out. The test conditions are summarized in Table 2. 
Table 2 Test conditions

\begin{tabular}{ccccc}
\hline Samples & Injection Pressure & Metallic Inserts & Tests & Quatity \\
\hline \multirow{5}{*}{$\# 1$} & OPSI & ZJ-A-1\#-1 ZJ-A-1\#-2 & Push-out/Breakaway torque & $1 / 1$ \\
\cline { 2 - 5 } & 60 PSI & ZJ-A-2\#-1 ZJ-A-2\#-2 & Push-out/Breakaway torque & $1 / 1$ \\
\cline { 2 - 5 } & 80 PSI & ZJ-A-3\#-1 ZJ-A-3\#-2 & Push-out/Breakaway torque & $1 / 1$ \\
\cline { 2 - 5 } & 1000 PSI & ZJ-A-4\#-1 ZJ-A-4\#-2 & Push-out/Breakaway torque & $1 / 1$ \\
\cline { 2 - 5 }$\# 2$ & 0 PSI & ZJ-B-1\#-1 ZJ-B-1\#-2 & Push-out/Breakaway torque & $1 / 1$ \\
\cline { 2 - 5 } & 60 PSI & ZJ-B-2\#-1 ZJ-B-2\#-2 & Push-out/Breakaway torque & $1 / 1$ \\
\cline { 2 - 5 } & 80 PSI & ZJ-B-3\#-1 ZJ-B-3\#-2 & Push-out/Breakaway torque & $1 / 1$ \\
\hline \multirow{3}{*}{$\# 3$} & 1000 PSI & ZJ-B-4\#-1 ZJ-B-4\#-2 & Push-out/Breakaway torque & $1 / 1$ \\
\cline { 2 - 5 } & 0 PSI & ZJ-C-1\#-1 ZJ-C-1\#-2 & Push-out/Breakaway torque & $1 / 1$ \\
\cline { 2 - 5 } & 60 PSI & ZJ-C-2\#-1 ZJ-C-2\#-2 & Push-out/Breakaway torque & $1 / 1$ \\
\cline { 2 - 5 } & 80 PSI & ZJ-C-3\#-1 ZJ-C-3\#-2 & Push-out/Breakaway torque & $1 / 1$ \\
\hline \multirow{3}{*}{$\# 4$} & 1000 PSI & ZJ-C-4\#-1 ZJ-C-4\#-2 & Push-out/Breakaway torque & $1 / 1$ \\
\cline { 2 - 5 } & 0 PSI & ZJ-D-1\#-1 ZJ-D-1\#-2 & Push-out/Breakaway torque & $1 / 1$ \\
\cline { 2 - 5 } & 60 PSI & ZJ-D-2\#-1 ZJ-D-2\#-2 & Push-out/Breakaway torque & $1 / 1$ \\
\cline { 2 - 5 } & 80 PSI & ZJ-D-3\#-1 ZJ-D-3\#-2 & Push-out/Breakaway torque & $1 / 1$ \\
\cline { 2 - 5 } & 1000 PSI & ZJ-D-4\#-1 ZJ-D-4\#-2 & Push-out/Breakaway torque & $1 / 1$ \\
\hline
\end{tabular}

The push-out test process is illustrated in Figure. 3. The computer-controlled electronic universal testing machine applies axial load on the metallic insert bonded into CFRP laminates via a mandrel until the metallic insert looses in axial direction.

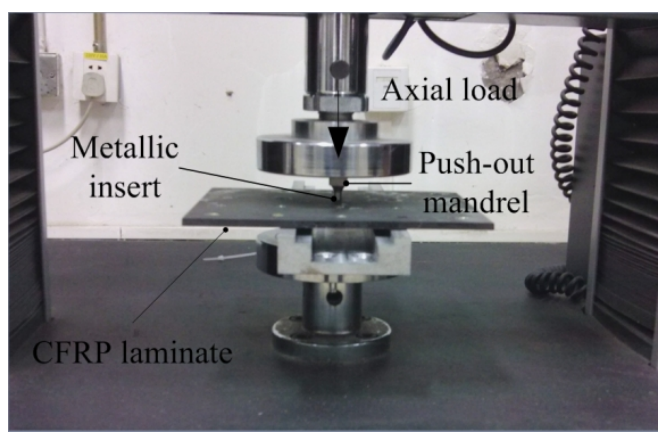

(a) Computer-controlled electronic universal testing machine Figure.3. Push-out test

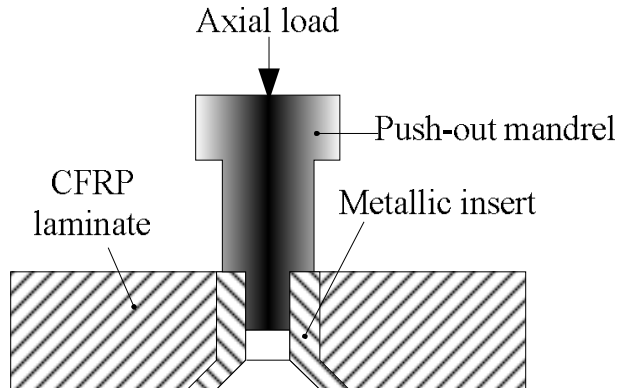

(b) Illustration of push-out test process

The breakaway torque test process is illustrated in Figure. 4. The torsion testing machine applies radial torque on the metallic insert bonded into CFRP laminates via a mandrel until the metallic insert looses in radial direction.

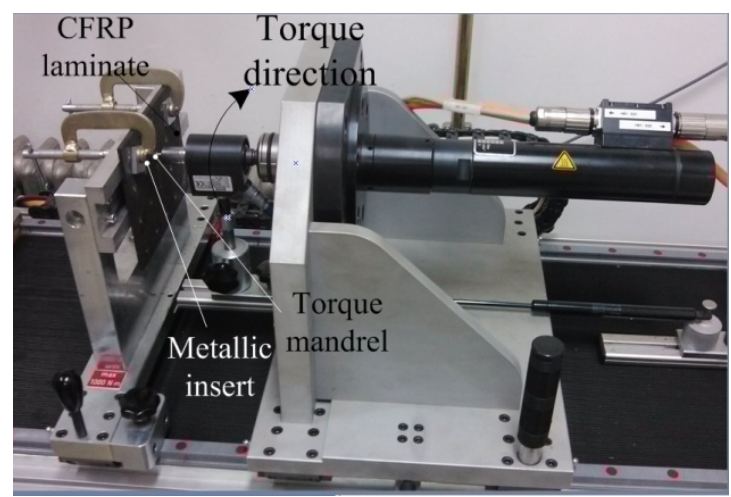

(a) Torsion testing machine

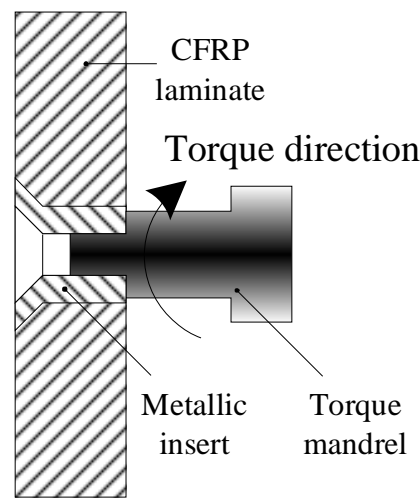

(b) Illustration of breakaway torque test process

Figure.4. Breakaway torque test 


\section{Experimental results and discussions}

Push-out test results and discussions. The push-out test results are showed in Figure. 5. The minimum push-out value of A type sample is $916 \mathrm{~N}$ and the maximum fluctuation in amplitude of push-out is $217 \mathrm{~N}$ under the four different types of injection pressure. The minimum push-out value of B type sample is $703 \mathrm{~N}$ and the maximum fluctuation in amplitude of push-out is $759 \mathrm{~N}$ under the four different types of injection pressure. The minimum push-out value of $\mathrm{C}$ type sample is $826 \mathrm{~N}$ and the maximum fluctuation in amplitude of push-out is $369 \mathrm{~N}$ under the four different types of injection pressure. The minimum push-out value of $\mathrm{D}$ type sample is $929 \mathrm{~N}$ and the maximum fluctuation in amplitude of push-out is $698 \mathrm{~N}$ under the four different types of injection pressure. Figure 6 shows the fluctuation in amplitude of push-out for $\mathrm{A}$ and $\mathrm{C}$ is less and the fluctuation in amplitude of push-out for B and D is greater. Generally, the smaller the installing hole is, the better the installing strength is. However, the results are not according to the regulation. Therefore, it is mainly attributed to the different mixed ratio for adhesive to cause different adhesive strength. Moreover, the installation precise of metallic inserts is playing a less important role in adhesive strength than that of the different mixed ratio for adhesive.

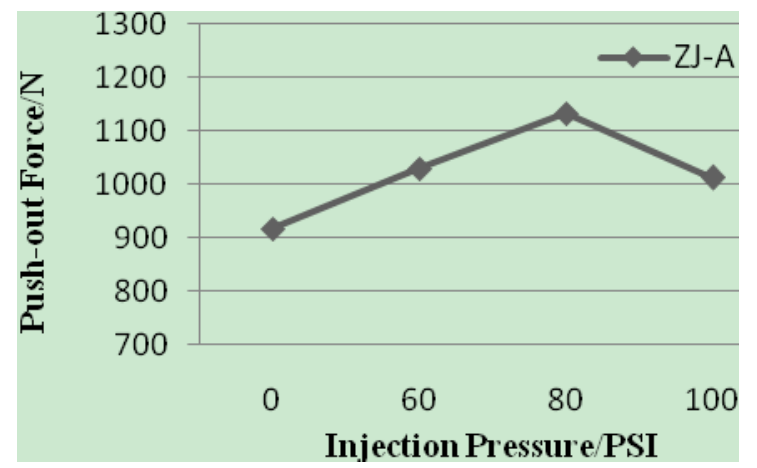

(a) Push-out force ZJ-A

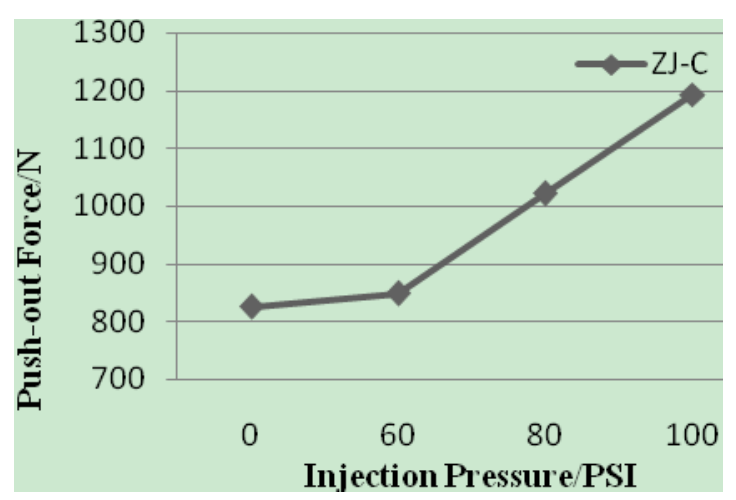

(c) Push-out force ZJ-C

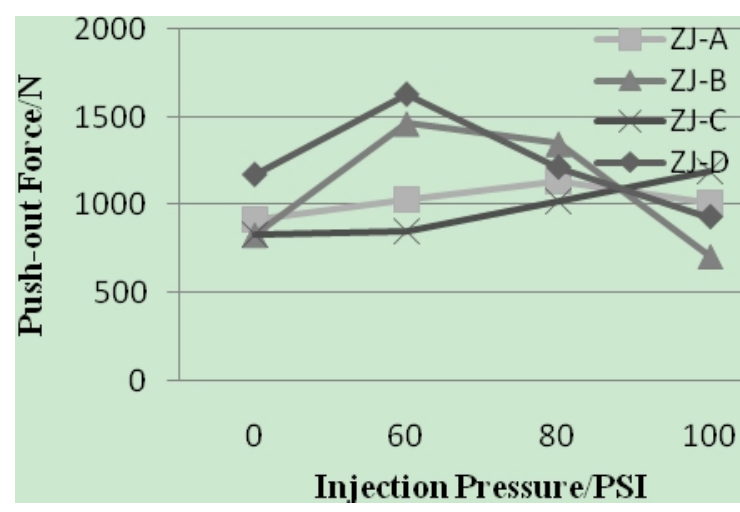

(e) Comparison of push-out force

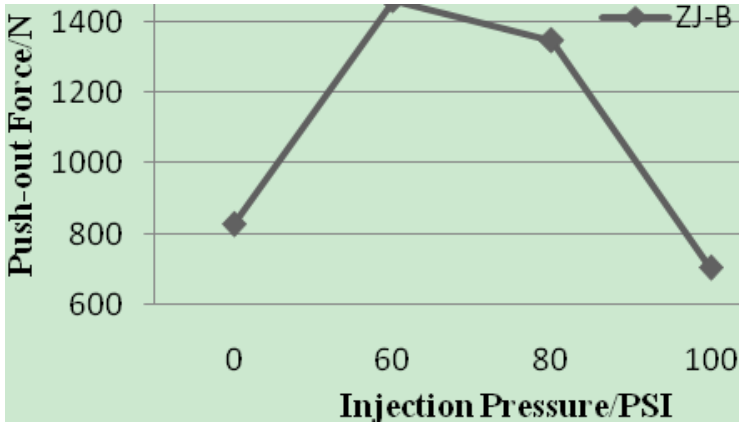

(b) Push-out force ZJ-B

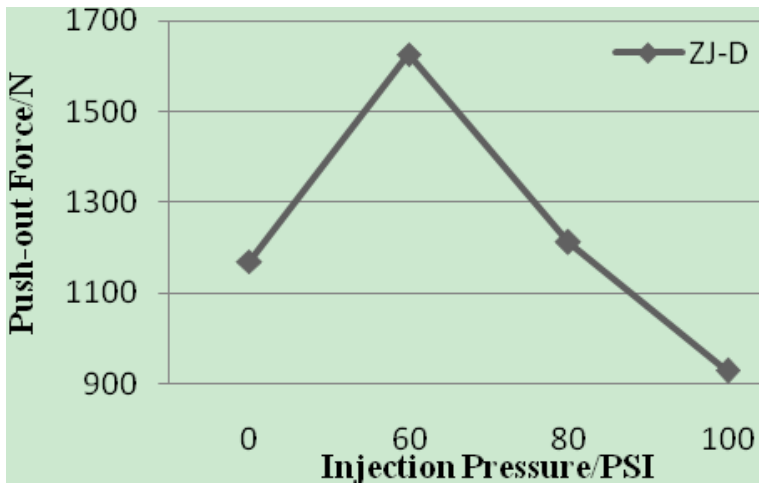

(d) Push-out force ZJ-D

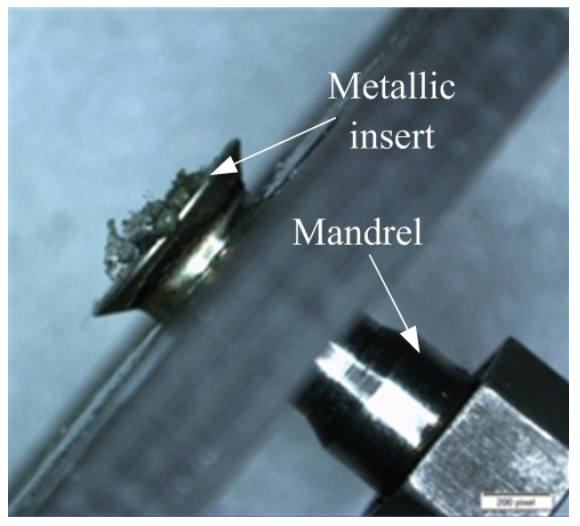

(f) Metallic insert pushed out

Figure.5. Push-out test 
Breakaway torque test results and discussions. Under the four different types of injection pressure, the breakaway torque test results are showed in Figure. 6. In the test, the threshold value of stopping breakaway torque is set for $4 \mathrm{~N}$.m. In these tests, all the inserts are not loosed. However, due to the strength of mandrels is not enough to loose the bonded metallic inserts, less of mandrels were broken when the torque value is less than $4.0 \mathrm{~N} . \mathrm{m}$. The minimum torque value of A type sample is $3.55 \mathrm{~N} . \mathrm{m}$. The minimum torque value of B type sample is $3.31 \mathrm{~N} . \mathrm{m}$. The minimum torque value of $\mathrm{C}$ type sample is $3.87 \mathrm{~N}$.m. The minimum torque value of $\mathrm{D}$ type sample is $3.86 \mathrm{~N} . \mathrm{m}$. The torque curves during test of four types of installing-hole are displayed in Figure 6(c). Because most of breakaway torque value can reach the threshold value, it is considered that the performance of bonded joint is stable.

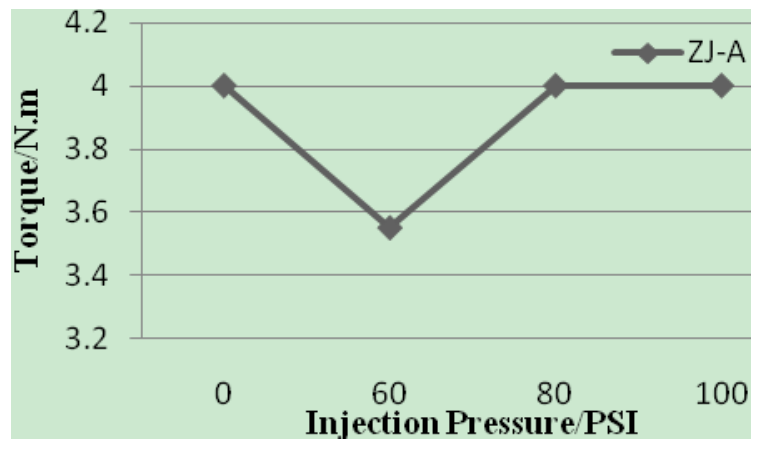

(a) Torque ZJ-A

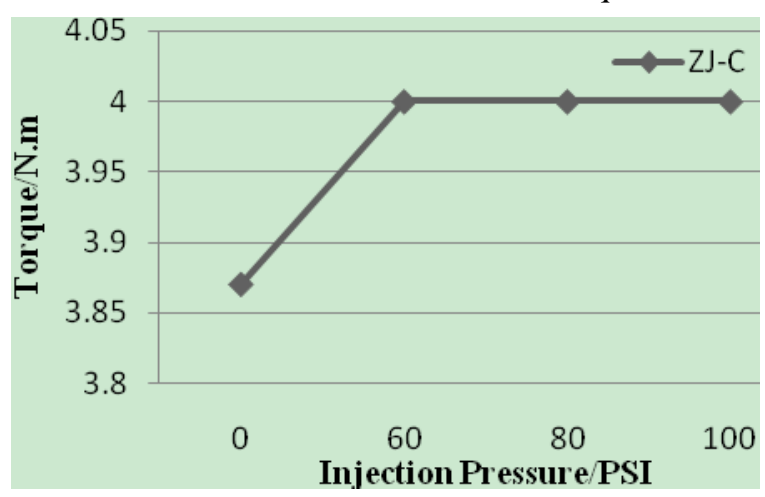

(c) Torque ZJ-C

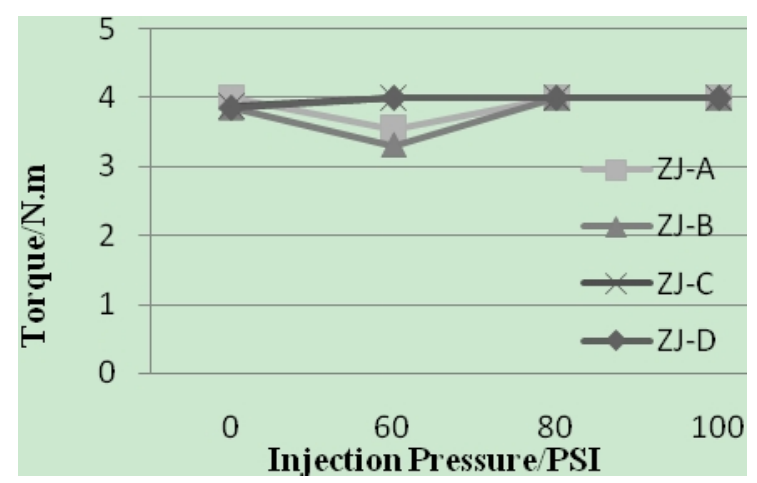

(e) Comparison of torque

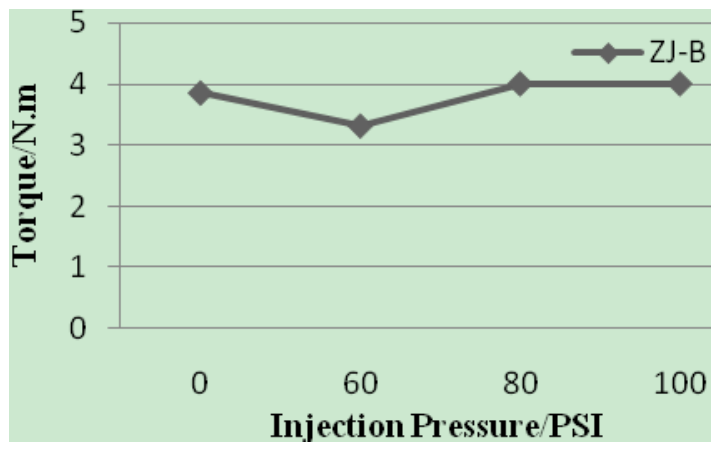

(b) Torque ZJ-B

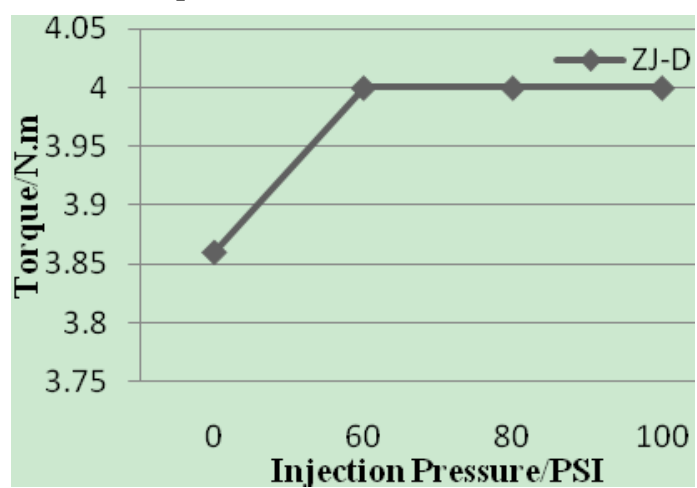

(d) Torque ZJ-D

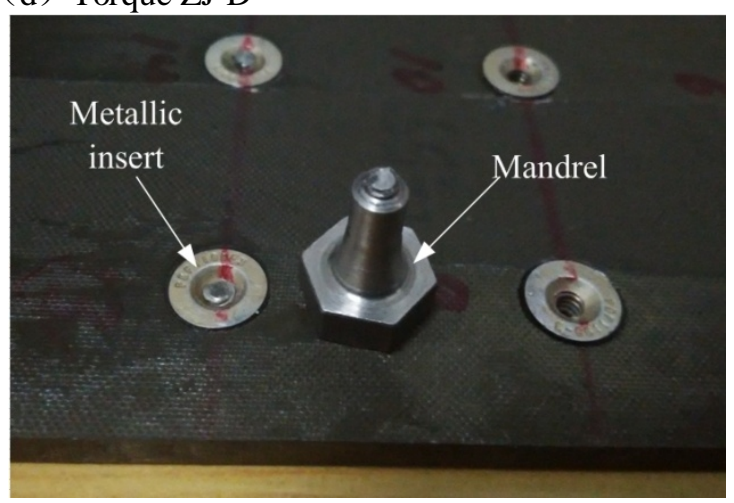

(f) Mandrel broken

Figure.6. Breakaway torque test

\section{Conclusions}

In this paper, a series of experiments were carried out to study the installing-holes parameter optimization for metallic inserts bonded into CFRP laminates. By performing the push-out and breakaway torque test for the four type installing-holes samples, the installing-holes parameter optimization experiments are studied. It can be concluded as follows: 
(1) For different type installing-holes, the push-out bearing capacity of metallic inserts bonded into CFRP laminates is not decreased obviously. The performance of bonded joint is stable.

(2) For different type installing-holes, the breakaway torque bearing capacity of metallic inserts bonded into CFRP laminates is not decreased significantly. The performance of bonded joint is stable.

(3)The joint strength of metallic inserts bonded into the CFRP laminates is affected larger by the adhesive property than that of the installation precise, so the adhesive parameters and installation process should be strictly controlled.

\section{References}

[1] Nilsson S.: submitted to J Compos Mater (1989).

[2] Herrera-Franco P, Cloud GL. : submitted to J Compos Mater (1994).

[3] Camanho, P. P., and F. L. Matthews.: submitted to Journal of Materials Design and Applications (2000)

[4] Camanho, P. P., et al.: submitted to Composites Part B: Engineering (2005)

[5] Brian Butler: submitted to 41st Structures, Structural Dynamics, and Materials Conference and Exhibit (2006) 